\title{
Materialism and Well-being in the UK and Chile: Basic Need Satisfaction and Basic Need Frustration as Underlying Psychological Processes
}

\author{
WENCESLAO UNANUE ${ }^{1,2 *}$, HELGA DITTMAR ${ }^{2}$, VIVIAN L. VIGNOLES ${ }^{2}$ and MAARTEN VANSTEENKISTE ${ }^{3}$ \\ ${ }^{1}$ Universidad Adolfo Ibáñez, Chile \\ ${ }^{2}$ University of Sussex, UK \\ ${ }^{3}$ University of Ghent, Belgium
}

\begin{abstract}
A growing body of evidence shows that materialistic values are linked to lower well-being. Selfdetermination theory offers an explanation through the low fulfilment of the basic psychological needs for autonomy, competence, and relatedness. However, recent research suggests that frustration of these psychological needs may also play an additional role. Using structural equation modelling in adult samples from an established massconsumer society ( $U K: N=958)$ and a fast-developing new economy (Chile: $N=257$ ) and employing more comprehensive measures to tap into a materialistic orientation than used in previous studies, we found that a materialistic value orientation related negatively to well-being and positively to ill-being and that both psychological need satisfaction and psychological need frustration played an explanatory role herein. The model was found to be highly equivalent across both samples, supporting the cross-cultural generality of the mechanisms involved. Copyright (C) 2014 European Association of Personality Psychology
\end{abstract}

Key words: consumer culture; materialistic values; life goals; well-being; ill-being; self-determination theory; need satisfaction; need frustration; adults

The endorsement of materialistic values in contemporary consumer cultures refers to the importance ascribed to the ownership and acquisition of material goods in achieving major life goals' (Richins, 2004, p. 210). Although the acquisition of more money and material goods is presented as a route to a successful and satisfying life, a growing body of research indicates that a materialistic value orientation is associated negatively with well-being (Dittmar, 2008; Kasser \& Ahuvia, 2002; Kasser \& Kanner, 2004). The vast majority of studies on materialism were conducted in North America and Western Europe, but there is preliminary evidence that a materialistic value orientation also relates to ill-being in non-mass-consumer societies such as Russia (Ryan et al., 1999) and India (Dittmar \& Kapur, 2011). Moreover, the explanatory (i.e. mediating) processes that underlie this link have received limited empirical attention in the research to date. In the current contribution, we tested the role of the satisfaction and frustration of the basic psychological needs for autonomy, competence, and relatedness, as conceived within self-determination theory (SDT; Deci \& Ryan, 2000), to account for the relationship between materialistic values and both well-being and ill-being, among adults from two fairly different societal contexts: that is, an established mass-consumer society (i.e. the UK) and a fast developing new economy (i.e. Chile).

*Correspondence to: Wenceslao Unanue, Universidad Adolfo Ibánez Business School, Diagonal Las Torres 2700, Edificio C (Postgrado), Peñalolén, Santiago, Chile.E-mail: wenceslao.unanue@uai.cl

\section{Materialistic values and well-being}

Past work on materialism has operationalized this construct in diverging ways, including society-level normative beliefs about the goals that a society should pursue (e.g. Inglehart, 2000, 2008; Inglehart \& Baker, 2000); personal attitudes and beliefs towards money (e.g. Tang, Kim, \& Tang, 2002; Tang, Tang, \& Luna-Arocas, 2005); measures of power values (e.g. Schwartz, 1992); and measures of personality traits such as envy, non-generosity and possessiveness linked to material possessions (e.g. Belk, 1985). However, as noted in a recent meta-analysis (Dittmar, Bond, Kasser, \& Hurst, in press), the most common approaches in the materialism literature have focused on personally internalized materialist values and beliefs (Richins \& Dawson, 1992), and extrinsic (versus intrinsic) life goals and aspirations (Kasser \& Ryan, 1993, 1996).

The materialistic values scale (Richins \& Dawson, 1992), widely used in consumer research, measures three key components: that is, acquisition centrality, success, and happiness. According to this perspective, the more people value material rewards as a central life goal, see them as a key route to achieve success and happiness, and use them to define their identities, the more materialistic they are (Richins \& Dawson, 1992). The Aspiration Index (Kasser \& Ryan, 1993, 1996), which has been more influential within mainstream personality and social psychology, assesses the importance a person places on extrinsic life goals (e.g. fame, image, and wealth), compared with intrinsic life goals (e.g. self-acceptance, affiliation, community involvement, and health). According to this perspective, the higher people 
rate the importance of extrinsic goals in comparison with intrinsic goals, the more materialistic they are. Thus, the Aspiration Index assesses the relative importance of a materialistic value orientation in a person's overall value system, whereas the materialistic values scale measures the endorsement of a materialistic value orientation as such (Dittmar, 2008).

According to both perspectives, materialism is a value system at the heart of consumer culture, which places strong emphasis on the acquisition of money, fame, success, and image, and which portrays a materialistic lifestyle as the ideal pathway to happiness and well-being (Kasser \& Kanner, 2004, Richins, 2004; Richins \& Dawson, 1992). Recent research (Twenge, Campbell, \& Freeman, 2012) indicates that younger generations (i.e. generation $\mathrm{X}$ and $\mathrm{Y}$ ) have adopted more strongly this value orientation than older generations (i.e. Babyboomers), and the mass media has presumably played a crucial role herein. From early childhood, people receive messages about the value of pursuing money, fame, and success, reinforcing the adoption of the ideals promoted by consumer culture (Dittmar, 2008).

Yet, numerous studies have reported that individuals with more materialistic values and aspirations show lower wellbeing, indicated by lower life satisfaction (e.g. Burroughs \& Rindfleisch, 2002, Vansteenkiste, Soenens, \& Duriez, 2008), self-actualization (e.g. Kasser \& Ryan, 1993, 1996), positive affect (e.g. Christopher \& Schlenker, 2004), vitality (e.g. Kasser \& Ahuvia, 2002), and happiness (e.g. Burroughs \& Rindfleisch, 2002; see Vansteenkiste et al., 2008, for an overview). As well as relating negatively to well-being, materialism has been found to relate positively to various indicators of ill-being, including negative affect (e.g. Christopher, Kuo, Abraham, Noel, \& Linz, 2004), alcohol and substance use (e.g. Williams, Cox, Hedberg, \& Deci, 2000), physical symptoms (e.g. Kasser \& Ryan, 1996), depressive symptoms (e.g. Vansteenkiste, Duriez, Simons, \& Soenens, 2006), and unhappiness (e.g. Kasser \& Ahuvia, 2002). This pattern of correlates has been further supported in a recent meta-analysis by Dittmar et al. (in press).

Dittmar et al. (in press) noted that existing work on materialism and well-being has focused on four broad categories of outcomes: The first and main category that received attention is subjective well-being (SWB). SWB is central to the hedonic (as opposed to the eudaimonic) approach to well-being (Gallagher, Lopez, \& Preacher, 2009; Keyes, Shmotkin, \& Ryff, 2002; Ryan \& Deci, 2001), which defines well-being in terms of attaining pleasure and avoiding pain (Ryan \& Deci, 2001). SWB 'refers to people's sense of wellness in their lives, in both thoughts and feelings' (Diener \& Tay, 2012, p. 1) and includes satisfaction with life as well as higher positive affect and lower negative affect (Diener, 1984). Therefore, it reflects both emotional experience and cognitive evaluations of people's lives, and it encompasses both positive (i.e. well-being) and negative (i.e. ill-being) dimensions. The second well-being category that has received attention in the materialism literature is self-appraisals, including measures related to self-esteem, positive and negative self-concept, self-doubt, selfambivalence, and self-discrepancies constructs. The third category is mental ill-health, most commonly indexed with measures of depressive symptoms and anxiety. The fourth category includes measures related to physical health, including measures of physical symptoms as well as health risk behaviours.

For the current research, we used an expanded version of Diener's (1984) three-dimensional conception of SWB, involving positive affect, negative affect, and life satisfaction. We enriched our assessment of emotional experience with the inclusion of measures of positive (i.e. vitality) and negative (i.e. depressive symptoms) mental health alongside the commonly used measures of positive and negative affect, and we added a fourth dimension focusing on symptoms of physical ill-health. Thus, we tested the predictive effects of materialistic values on two dimensions of well-being (emotional well-being and life satisfaction) and two dimensions of ill-being (emotional ill-being and physical symptoms of ill-health).

\section{Psychological need satisfaction and psychological need frustration}

An important lacuna in the present materialism literature is that it is not yet well understood why materialism relates to both lower well-being and higher ill-being. Therefore, grounded in SDT-and more specifically in goal content theory, one of SDT's five mini-theories (Ryan \& Deci, 2000; Vansteenkiste, Niemiec, \& Soenens, 2010)—we examined whether the satisfaction and frustration of the psychological needs for autonomy, competence, and relatedness would help to account for this link.

Within SDT (Deci \& Ryan, 2000; Ryan \& Deci, 2000), it is maintained that human beings' growth and well-being is fostered by the satisfaction of basic psychological needs for autonomy, competence, and relatedness. Autonomy refers to the need to choose one's own life direction and to experience a sense of psychological freedom in one's behaviour; competence refers to the need to reach one's goals and to effectively carry out one's daily activities; relatedness refers to the need to develop intimate and close relationships with others and to feel part of a group. Satisfaction of these needs plays a central role in promoting well-being (Ryan \& Deci, 2001). Diverse forms of research have convincingly shown that the fulfilment of these psychological needs relates to SWB, positive self-appraisals, and the absence of mental and physical ill-health (Deci \& Ryan, 2000; Vansteenkiste et al., 2010). Importantly, the link between need satisfaction and well-being has been found in both Eastern and Western cultures (e.g. Chirkov, Ryan, \& Sheldon, 2011; Chirkov, Ryan, \& Willness, 2005; Church et al., 2013; Deci et al., 2001; Sheldon, Elliot, Kim, \& Kasser, 2001; Ryan et al., 1999; Taylor \& Lonsdale, 2010) and using both self-reports as well as external ratings of well-being (e.g. Ahmad, Vansteenkiste, \& Soenens, 2013).

More recently, increasing empirical attention has been devoted to the topic of need frustration (Bartholomew, Ntoumanis, Ryan, Bosch, \& Thøgersen-Ntoumani, 2011). According to recent theorizing, the relation between need satisfaction and need frustration is said to be asymmetrical 
(Vansteenkiste \& Ryan, 2013). That is, although the lack of need fulfilment does not necessarily entail the experience of need frustration, need frustration by definition implies that the fulfilment of the needs becomes blocked. To illustrate, when an employee does not feel very connected to his colleagues, this does not necessarily entail that he or she feels excluded by them. Yet, when he or she is excluded from a social event or meeting, it is certain that he or she will not feel connected to them. Thus, different from low need fulfilment, need frustration involves the more active obstruction of the psychological needs. In line with this theorizing, recent studies have shown that need satisfaction and need frustration represent distinct factors (e.g. Cordeiro, Paixão, Lens, \& Silva, 2013; Sheldon, 2011; Sheldon, Abad, \& Hinsch, 2011; Sheldon \& Gunz, 2009; Sheldon \& Hilpert, 2012). For example, Sheldon and Gunz (2009) found that the frustration of the psychological needs is associated with a higher desire to reduce need frustration, but that need satisfaction does not reduce the desire to satisfy the needs. Sheldon et al. (2011) studied the association between the use of Facebook, and the satisfaction and dissatisfaction of the basic psychological needs, finding that frequency of Facebook usage was correlated positively with both satisfaction and frustration of the relatedness need.

Further, it is maintained that whereas need satisfaction is critical for growth to take place, need frustration would awaken our vulnerabilities and relate to maladjustment (Bartholomew et al., 2011; Vansteenkiste \& Ryan, 2013). In line with this claim, need satisfaction was found to relate especially to positive outcomes, such as vitality and positive affect (e.g. Stebbings, Taylor, Spray, \& Ntoumanis, 2012), whereas need frustration related to maladaptive outcomes, including burnout, disordered eating, and elevated levels of objectively assessed immunological problems. The differential role of need frustration was not only found at the betweenperson but also at the within-person level, with daily fluctuation in psychological need frustration (but not in need satisfaction) relating to daily variation in bulimic symptoms (Verstuyf, Vansteenkiste, Soenens, Boone, \& Mouratidis, 2013).

The needs for autonomy, competence, and relatedness are thought to intervene in the materialism-well-being association. Indeed, Kasser and Ryan $(1993,1996)$ argued that the pursuit of intrinsic, relative to more extrinsic and materialistic, goals would help to fulfil these needs, thereby promoting individuals' well-being. That is, focusing on intrinsic aspirations such as helping others in need, building good bonds, or developing one's skills promotes greater task absorption, which, in turn, facilitates greater skill development (Mouratidis, Vansteenkiste, Lens, Michou, \& Soenens, 2013; Vansteenkiste et al., 2004). In contrast, the pursuit of extrinsic goals prompts the engagement in social comparison processes, which can be stressful and socially alienating (Banerjee \& Dittmar, 2008; Dittmar et al., in press). Thus, whereas intrinsic goal pursuit may provide greater opportunities for need satisfaction, the pursuit of extrinsic goals may interfere with need satisfaction and even elicit experiences of need frustration.

A few previous studies have explored the explanatory role of basic psychological need satisfaction in the link between intrinsic, relative to extrinsic, goals and different aspects of well-being in diverse life domains. For instance, Vansteenkiste et al. (2007) found that holding an intrinsic, relative to an extrinsic, work value orientation was associated positively with well-being because of the fulfilment of psychological needs. In the exercise domain, Sebire, Standage, and Vansteenkiste (2009) found that need satisfaction could partially account for the relation between intrinsic, relative to extrinsic, exercise goals and psychological well-being. Finally, Thøgersen-Ntoumani, Ntoumanis, and Nikitaras (2010) reported that a higher focus on intrinsic goals such as health associated positively with basic need satisfaction, which in turn, was negatively associated with unhealthy weight-management behaviours.

The present study aimed to extend this small body of literature in four ways. First, past work on intrinsic and extrinsic goal contents and needs failed to take the role of need frustration explicitly into account, as the items used to assess need satisfaction tapped into the experience of low satisfaction (e.g. 'I often do not feel very capable') rather than the experience of active need frustration (e.g. 'I often experience some kind of failure'). Therefore, the present research examined the unique roles of psychological need satisfaction and need frustration in the relation between intrinsic and extrinsic aspirations and both well-being and ill-being. Preliminary evidence for the role of need frustration was provided by Verstuyf, Vansteenkiste, and Soenens (2012), who showed that need frustration could account for the link between dieting to achieve physical attractiveness and bulimic symptoms. Yet, these authors did not assess need satisfaction, leaving open the question whether both need satisfaction and need frustration play a critical role.

Second, all studies presented earlier were grounded in the SDT tradition, thus being limited by relying on intrinsicextrinsic goal measures. Yet, it seems important to explore whether need satisfaction and need frustration can also account for the link between materialistic values or beliefs (Richins \& Dawson, 1992) and well-being. Further, we believe it is unfortunate that the fields of materialism and SDT have been developed fairly independently in spite of their clear points of convergence. Only one previous study known to us (Van Hiel, Cornelis, \& Roets, 2010) has attempted to bridge this gap, tapping into both adolescents' materialistic value orientation (Richins \& Dawson, 1992) and their intrinsic and extrinsic aspirations (Kasser \& Ryan, 1993, 1996).

A third novel aspect is that the present study focused on participants' aspirations and materialistic strivings in their lives in general rather than vis-à-vis specific contexts (work, sports, and eating behaviour), as was the case in the previously mentioned studies. Thus, we believe that our study is the first to examine whether need satisfaction and need frustration can account for the materialism-well-being association in more general terms.

Fourth, most previously published research into materialism and well-being has focused on relatively affluent nations (Dittmar et al., in press). As we discuss shortly, some 
theoretical perspectives imply that the negative link between materialism and well-being might be weaker or even reversed in developing nations (e.g. Inglehart, 2000; Inglehart \& Baker, 2000). Very few previous studies have tested the correlates of materialism in non-mass-consumer societies (for exceptions, see Dittmar \& Kapur, 2011; Ryan et al., 1999), and we are aware of no published research that has examined these links in any South American nation. Hence, we tested here whether materialism yields the same well-being correlates in a developing South American nation (Chile) as in a developed Western European consumer society (the UK).

\section{Contexts for the present research: UK and Chile}

In this research, we tested parallel predictions among adults in the UK and Chile. Recent cross-national evidence suggests that materialistic values may yield a different pattern of correlates across cultures (Gatersleben, Jackson, Meadows, Soto, \& Yan, 2012; Schaefer, Hermans, \& Parker, 2004). For instance, Gatersleben et al. (2012) studied to what extent materialism was associated with different environmental outcomes among 16- to 25-year-olds in the UK, Spain, and China. Although the results were fairly similar in the UK and Spain, they were quite different for Chinese young adults. Materialism was negatively associated with environmental worldviews and ecologically responsible behaviour intentions in the UK and in Spain, but not in China. Such findings raise the question as to whether the adoption of materialistic values will have similar or different correlates in the UK and Chile, in terms of maladjustment and non-optimal functioning. We should acknowledge that our choice to sample participants in these two nations was mainly pragmatic, on the basis of the nationalities of the primary researchers. Nonetheless, we believe that it is valuable to compare findings across UK and Chilean samples for a number of reasons.

First, data on each country's gross domestic product (GDP) per capita show that the UK GDP per capita based on purchasing power parity was almost $50 \%$ higher than in Chile in the period between 2008 and 2012 (World Bank, 2013). Notably, different theoretical perspectives offer contradictory predictions regarding the possible moderating impact of national affluence on the materialism-well-being relationships. Goal attainment perspectives suggest that materialistic individuals living in poorer countries would be less able to fulfil their desires (Dittmar et al., in press). Therefore, we could expect that the negative association of materialism and well-being may be smaller among those living in a more affluent country such as the UK than among those living in a less affluent country such as Chile. On the other hand, a Maslovian perspective suggests that striving for materialistic rewards may be more adaptive in an economic context that provides less financial security to its members than in a more developed context where basic physiological needs are more likely to be met (e.g. Inglehart \& Baker, 2000; Oishi, Diener, Lucas, \& Suh, 1999). Following this argument, one could expect stronger negative associations between materialism and well-being in the UK than in Chile. ${ }^{1}$

Second, Chile has been classified recently as the most unequal among the 34 OECD countries (OECD, 2013). The Gini coefficient, a standard measure of income inequalities, is almost 44\% higher in Chile than in the UK. Research has shown that the link between a materialistic values orientation and personal well-being is significantly moderated by the extent of income inequalities in a country, which may be linked to social comparison processes (Dittmar et al., in press). Therefore, to analyse how materialism relates differently to well-being in countries with different levels of inequalities is an interesting question to explore.

Third, the two countries show marked differences on a variety of indicators of consumer culture penetration-which has been viewed as a key element in the formation of materialistic value priorities (Dittmar, 2008; Kasser \& Kanner, 2004). According to the Human Development Report (United Nations, 2010), in 2008, the mobile and land lines subscriptions rate per 100 people was 180 in the UK, but just 109 in Chile. ${ }^{2}$ In the same period, internet users and broadband subscriptions per 100 inhabitants were respectively 76 and 28.2 in the UK but just 32.5 and 8.5 in Chile. Only $19 \%$ of Chileans owned a debit card in 2011 (SBIF, 2011), compared with virtually all UK residents in 2010 (Dittmar \& Kapur, 2011). Differences were less pronounced for credit cards ownership: $41 \%$ in Chile in 2008, compared with $68 \%$ in the UK. These differences in consumer cultural penetration provide another reason that materialistic values could potentially lead to different wellbeing outcomes in the UK and Chile.

In summary, the UK and Chile vary in a number of respects (e.g. economic wealth, income inequalities, and consumer culture penetration), thereby providing an initial opportunity to test our hypothesized associations in a developing nation and to compare the results with an established mass-consumer society. By doing so, we would be able to examine whether the hypothesized correlates of materialism could be generalized to Chile.

\section{Summary of aims and predictions}

Although the seminal papers on people's materialistic strivings (Richins \& Dawson, 1992) and their extrinsic, relative to,

\footnotetext{
${ }^{1}$ The literature also yields contradictory predictions regarding the effect of economic development on the adoption of materialistic values. Recen meta-analyses by Twenge and colleagues (Twenge, 2010; Twenge et al., 2012; Twenge \& Kasser, 2013) suggest that materialistic values are rising across generations in contemporary mass-consumer societies, a trend that some authors have attributed to increased exposure to advertising that shows extrinsic life goals as the pathways to happiness and well-being (Kasser \& Ryan, 1993, 1996). On the other hand, extensive cross-cultural research by Inglehart and colleagues (e.g. Inglehart, 2000, 2008; Inglehart \& Baker, 2000) suggests that increasing economic development is accompanied by a shift from materialist (survival) towards post-materialist (self-expression) values. The difference between these findings likely lies in the very different ways that these groups of researchers operationalized materialistic values - in terms of both the level of explanation (societal norms vs. personally internalized life goals and values) and the content of the beliefs (concern for economic and physical security vs. believing that material rewards are the route to well-being). However, our current goal was not to test for mean differences in values across the two samples in our study but to examine whether individual differences in these values within each of the two samples have similar or different associations with need satisfaction and frustration and with well-being and ill-being.

${ }^{2}$ One person may have more than one subscription.
} 
their intrinsic aspirations (Kasser \& Ryan, 1993) came out about two decades ago and have spurred dozens of studies over the years, few studies to date have made use of comprehensive measures, instead making use of either the materialistic values scale or the Aspiration Index. In two relatively large samples of adults, we assessed both participants' materialistic value orientations and their intrinsic and extrinsic aspirations. Further, although the materialism-wellbeing association is well-documented, the reasons that materialistic strivings interfere with growth and even elicit ill-being remain less well understood. Consistent with recent developments within SDT (Bartholomew et al., 2011; Vansteenkiste \& Ryan, 2013), we examined the separate and unique roles of need satisfaction and need frustration as potential mediators of the link from materialism to well-being and ill-being. Following Vansteenkiste and Ryan (2013), we predicted that need satisfaction would be primarily associated with growth and wellness, and need frustration primarily with malfunctioning and ill-being, but we also expected to observe weaker crosspaths: need satisfaction may play a protective role against malfunctioning and ill-being, and need frustration may lead to lower positive well-being because individuals have developed fewer resources for growth. Finally, we tested whether the paths in our model were moderated by national context, comparing adult samples in the UK - an established mass-consumer society-with Chile_-a fast-growing new economy, where no previous research to our knowledge has been conducted into materialistic values or into SDT.

On the basis of the literature reviewed earlier, we sought to test the following hypotheses. First, we predicted that a stronger materialistic value orientation (modelled by the Aspiration Index and the materialistic values scale) would be linked negatively to well-being and positively to ill-being (Hypothesis 1). Next, we aimed to test the integrated model depicted in Figure 1, in which the link between materialism and well-being would be explained by both psychological need satisfaction and psychological need frustration. This model was tested in a gradual and stepwise fashion. Specifically, we began by examining whether relationships between materialism and well-being and ill-being could be partially explained by basic need satisfaction (Hypothesis 2). We expected only partial mediation as the contribution of materialism to ill-being may not just be accounted for by the absence of need satisfaction but also requires the more active obstruction and, hence, frustration of the psychological needs. Therefore, in a next step, we examined whether the addition of basic need frustration to our model would help to fully account for the materialism-ill-being association

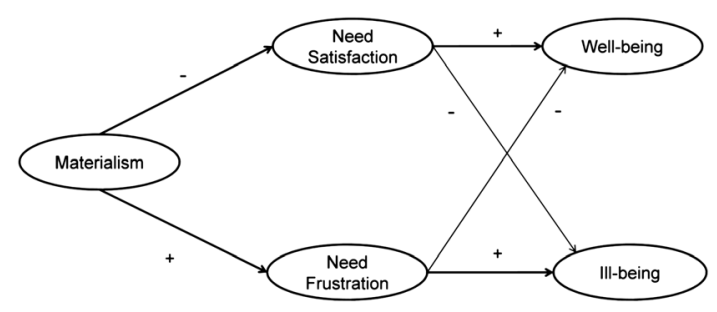

Figure 1. Theoretical model for the associations between materialism, need satisfaction, need frustration, well-being, and ill-being in the UK and Chile. and thus testify to the incremental role of need frustration (Hypothesis 3). Finally, given the claim of SDT to study universal psychological processes (Deci \& Ryan, 2000), we expected to find comparable support for $\mathrm{H} 1$ to $\mathrm{H} 3$ in samples drawn from both UK and Chilean contexts (Hypothesis 4).

\section{METHOD}

\section{Participants and procedure}

The British sample consisted of 958 adults living in the UK, ranging in age from 20 to 77 years $($ Mean $=44.68 ; S D=13.98)$. Respondents (59\% female) were former graduates, recruited through the alumni office of a university in the South East of England. The Chilean sample consisted of 257 adults living in Chile, ranging in age from 19 to 71 years (Mean $=34.81$; $S D=10.54)$. Respondents (53\% female) were also all former graduates, recruited mostly through the alumni office of a university in Santiago and also through personal contacts of the first author. Using a sample that consists entirely of graduates has the advantage that educational level is controlled for.

Statistical analyses revealed that the two samples differed significantly in age $(F[1,1214], p<.001)$, but not in gender distribution, $\chi^{2}(1)=2.94, p=.09$. Nonetheless, controlling for these background characteristics did not change the substantive results reported in our structural models.

In a first instance, participants were sent an introductory email containing a brief description of the study along with a web link to the survey. UK participants were invited to participate in a research project by completing an online survey and were offered entry into a prize draw for university memorabilia. In Chile, the same instructions were sent, but participants were not offered entry into a prize draw.

The study was conducted according to British Psychological Society and American Psychological Association guidelines. All participants provided written consent and were informed that they could withdraw from the study at any point. The purpose of the research was described in broad terms (hence, no deception was involved), and respondents were given the opportunity to receive a summary of the research findings. Respondents were also asked whether they would agree to participate in future research, as it was anticipated that the survey would be the first wave of a multi-wave project.

The first page of the survey contained a brief description of the study, and the second page informed participants of their right to withdraw at any time, as well as assuring their anonymity and confidentiality with regard to their responses. Then, participants were asked to complete the core measures for the present research: well-being, need satisfaction, need frustration, and materialism. Some other measures were collected that are not relevant to the present hypotheses (e.g. measures about environmental attitudes and behaviours). The final section of the survey assessed demographic details, including (among others) age, gender, and income. This project used various scales, the majority of which have been used in previous research, and are known to have good psychometric properties. The questionnaire was translated into Spanish for the Chilean participants, and equivalence 
of meaning with the English version was checked through established back-translation procedures (Brislin, 1970).

In order to test for order effects, two versions of the survey were created. The first version asked participants first about well-being, need satisfaction, and need frustration and then about materialism. The second version of the survey asked participants first about materialism, and then about well-being, need satisfaction, and need frustration. Multivariate analyses of variance were carried out in each sample with version as the independent variable and all core construct measures as dependent variables. In both the UK $(F[10,947]=1.10, p=.23)$ and Chile $(F[10,246]=1.40$, $p=.18)$, the multivariate result was non-significant, indicating that the order in which respondents completed the measures did not have an impact on their responses.

\section{Measures}

\section{Materialism}

Materialism was modelled as a latent variable in the tested structural models, using indicators derived from the two most commonly used scales in the research literature (Dittmar et al., in press): the Aspiration Index (Kasser \& Ryan, 1993, 1996) and the materialistic values scale (Richins and Dawson, 1992).

Aspiration Index. We used a shortened, 30-item version of the Aspiration Index developed by Kasser and Ryan (1993, 1996) to assess the importance of different life goals. We used six categories of aspirations with five specific items within each category. Aspirations are either extrinsic (money, fame, and image) or intrinsic (affiliation, community involvement, and self-development). We asked people to rate how important each goal is to them personally from not at all (1) to very (7). Example items are To be a very wealthy person (money), To have my name known by many people (fame), To successfully hide the signs of ageing (image), To grow and learn new things (self-development), To have good friends that I can count on (affiliation), and To work for the betterment of society (community involvement). The internal reliability of the three intrinsic aspirations (self-development, community involvement, and affiliation) ranged from acceptable to good in the UK $\left(\alpha_{\mathrm{s}}=.67, .91\right.$, and .85 , respectively) and in Chile $\left(\alpha_{\mathrm{s}}=.76, .89\right.$, and .77 , respectively). The internal reliability of the three extrinsic aspirations (money, fame, and image) was good in the UK $\left(\alpha_{\mathrm{s}}=.86, .86\right.$, and .82 , respectively) and in Chile $\left(\alpha_{\mathrm{s}}=.87, .90\right.$, and .85, respectively).

Materialistic values scale. We used the shortened, nineitem version developed by Richins and Dawson (1992) and revised by Richins (2004). Example items are I admire people who own expensive homes, cars, and clothes, Buying things gives me a lot of pleasure, and My life would be better if I owned certain things I don't have. Participants rated these statements on a 6-point Likert-type scale ranging from strongly disagree (1) to strongly agree (6). The internal reliability of the scale was good, both in the UK $(\alpha=.83)$ and in Chile $(\alpha=.81)$.

\section{Need satisfaction and need frustration}

To assess need satisfaction and frustration, we used a balanced measure developed by Sheldon and Gunz (2009). This scale consisted of 18 items; that is, six items per need, of which three tapped into need satisfaction and three tapped into need frustration. Psychometric properties, as well as distinctiveness between satisfaction and frustration, have been demonstrated by Sheldon and Hilpert (2012) and Cordeiro et al. (2013).

Basic psychological need satisfaction scale. This is a nineitem measure yielding a global score of individuals' need satisfaction including three items each to measure satisfaction of needs for autonomy, competence, and relatedness. Examples of items are as follows: I felt a sense of contact with people who care for me, and whom I care for (relatedness satisfaction); I was successfully completing difficult tasks and projects (competence satisfaction); and My choices expressed my 'true self' (autonomy satisfaction). Participants rated these statements on a 6-point Likert-type scale ranging from strongly disagree (1) to strongly agree (6). The internal reliability in this research was good, both in the UK $(\alpha=.85)$ and in Chile $(\alpha=.83)$.

Basic psychological need frustration scale. This is a nineitem measure yielding a global score of individuals' need frustration, including three items each to measure frustration of needs for autonomy, competence, and relatedness. Examples items are as follows: I was lonely (relatedness frustration); I experienced some kind of failure, or was unable to do well at something (competence frustration); and There were people telling me what I had to do (autonomy frustration). Participants rated these statements on a 6-point Likert-type scale ranging from strongly disagree (1) to strongly agree (6). The final scale showed a good internal reliability, both in the UK $(\alpha=.81)$ and in Chile $(\alpha=.79)$.

\section{Well-being}

We employed six scales assessing a wide array of well-being and ill-being dimensions, including measures typically used to assess SWB (life satisfaction, positive affect, and negative affect), as well as mental health measures (depressive symptoms and subjective vitality), and physical symptoms of illhealth. ${ }^{3}$ We used these measures to model two dimensions of well-being, life satisfaction, and emotional well-being (combining positive affect and vitality), and two dimensions of illbeing, emotional ill-being (combining negative affect and depressive symptoms), and physical symptoms of ill-health.

Satisfaction with life. We used the five-item Satisfaction with Life Scale developed by Diener, Emmons, Larsen, and Griffin (1985) to measure the cognitive component of SWB. Examples items are In most ways my life is close to my ideal and The conditions of my life are excellent. Participants rated these statements in a 6-point Likert-type scale ranging from strongly disagree (1) to strongly agree

\footnotetext{
${ }^{3}$ There are several existing measures of eudaimonic well-being in the literature (e.g. Ryff, 1989; Waterman et al., 2010). However, we decided not to use these scales in order to avoid excessive overlap with our need satisfaction and frustration measures.
} 
(6). The internal reliability of the scale was good, both in the UK $(\alpha=.87)$ and in Chile $(\alpha=.87)$.

Positive and negative affect. We used the 10-item measure International Positive and Negative Affect Schedule Short Form (Thompson, 2007), which includes separate subscales measuring positive affect (five items) and negative affect (five items). Example items asked participants how frequently they have felt inspired, alert, upset, and nervous during the last month. Participants rated these questions in a 5-point scale ranging from never (1) to always (5). The measure showed good internal reliability, both in the UK (positive affect: $\alpha=.79$; negative affect: $\alpha=.77$ ) and in Chile (positive affect: $\alpha=.72$; negative affect: $\alpha=.72$ ).

Vitality. We employed the Subjective Vitality scale (Ryan \& Frederick, 1997), a seven-item measure developed to evaluate how alive and alert people have been feeling during the last month. Participants rated their agreement with statements such as I feel alive and vital and I don't feel very energetic, on a 7-point response scale from not at all true (1) to very true (7). Internal reliability was good, both in the UK $(\alpha=.91)$ and in Chile $(\alpha=.87)$.

Depressive symptoms. We employed a slightly shortened version (16 items) of the Centre for Epidemiological Studies-Depression Inventory (Radloff, 1977) measure, designed to obtain an individual global depression score in nonclinical samples. Example items are I did not feel like eating, My appetite was poor, and I felt that I couldn't stop feeling down even with help from my family or friends. Participants rated these statements on a 4-point scale ranging from rarely or none of the time (0) to most or all of the time (3). The internal reliability of the scale was good, both in the UK $(\alpha=.89)$ and in Chile $(\alpha=.89)$.

Physical symptoms. We employed seven items from the General Health Questionnaire developed by Goldberg et al. (1997) to obtain a measure of participants' global health. For the purpose of this study, just part A ( 7 items) of the original scale was used, in order to focus on physical symptoms. Participants responded to questions such as how often they had recently been feeling in need of a good tonic or feeling run down and out of sorts on a 5-point scale from never (1) to always (5). The internal reliability of these seven items was good, both in the UK $(\alpha=.84)$ and in Chile $(\alpha=.81)$.

\section{RESULTS}

\section{Plan of analysis}

We employed structural equation modelling (AMOS 18.0 (Amos Development Corporation, Florida, U.S.A.)) to assess the hypothesized associations between materialism, need satisfaction, need frustration, and positive and negative wellbeing. Descriptive statistics and inter-correlations for these study variables are shown in Table 1 . On the basis of the recommendations of $\mathrm{Hu}$ and Bentler (1999) and Kline (2005), we assessed model fit using the root mean square error of approximation (RMSEA) and comparative fit index (CFI). Although cut-off values for these indices are often debated, we combined the recommendations of these authors and interpreted values of RMSEA $<0.06$ (or $<0.08$ ), and $C F I>0.95$ (or $>0.90$ ) as evidence of good (or acceptable) fit.

In order to proceed with the analysis, we modelled all constructs as latent variables using three, four, or six observed indicators per factor. Following the recommendations of Little, Cunningham, and Shahar (2002), for most indicators, we created item parcels, as described later.

\section{Materialism}

We modelled our materialism measure as a latent construct using six different indicators: three parcels provided by the Aspiration Index and three subscales of the materialistic values scale.

Aspiration Index. To obtain the relative importance placed on extrinsic aspirations compared with intrinsic ones, we followed Duriez, Vansteenkiste, Soenens, and De Witte (2007). First, an individual's overall mean score was subtracted from each individual score. Second, the intrinsic items were reversed, and an overall extrinsic versus intrinsic (E/I) value score was computed by averaging the extrinsic and (reversed) intrinsic scales. Cronbach's alpha ranged from acceptable in the UK $(\alpha=.73$, Mean $=-1.51, S D=0.42)$ to good in Chile $(\alpha=.82$, Mean $=-1.40, S D=0.36)$. Positive (negative) scores reflect a tendency to prefer extrinsic (intrinsic) rather than intrinsic (extrinsic) values. Finally, we randomly created three parcels by employing one extrinsic scale and one (reversed) intrinsic scale to be used as indicators.

Materialistic values scale. The materialistic values scale (Richins and Dawson, 1992) is based on the conceptualization of a value system with three interrelated dimensions: centrality, happiness, and success. On the basis of this, we created three indicators from the three subscales previously mentioned.

\section{Need satisfaction and need frustration}

We built latent variables for the need satisfaction and the need frustration scales, using three item parcels as indicators of each construct (Little et al., 2002). In order to give equal importance in our measures to the three basic needs, each parcel included items referring to all the three needs.

\section{Well-being}

Given our expectation that the need satisfaction scale would be more predictive of positive well-being outcomes whereas the need frustration scale would be more predictive of negative well-being outcomes, we initially tried to model positive and negative well-being as two latent constructs. However, initial analyses suggested that a two-factor model was too simple and that the six well-being variables would be better represented as four latent factors, two positive and two negative. Vitality and positive affect loaded together and were modelled as the first positive well-being latent construct: We created four item parcels (two from each scale) and called this emotional wellbeing. The second positive well-being latent construct was life satisfaction, which was modelled using three item parcels. Negative affect and depressive symptoms loaded together and were modelled as the first ill-being construct: We created four item parcels (two from each scale) and called this emotional ill-being. The second negative well-being variable focused on 


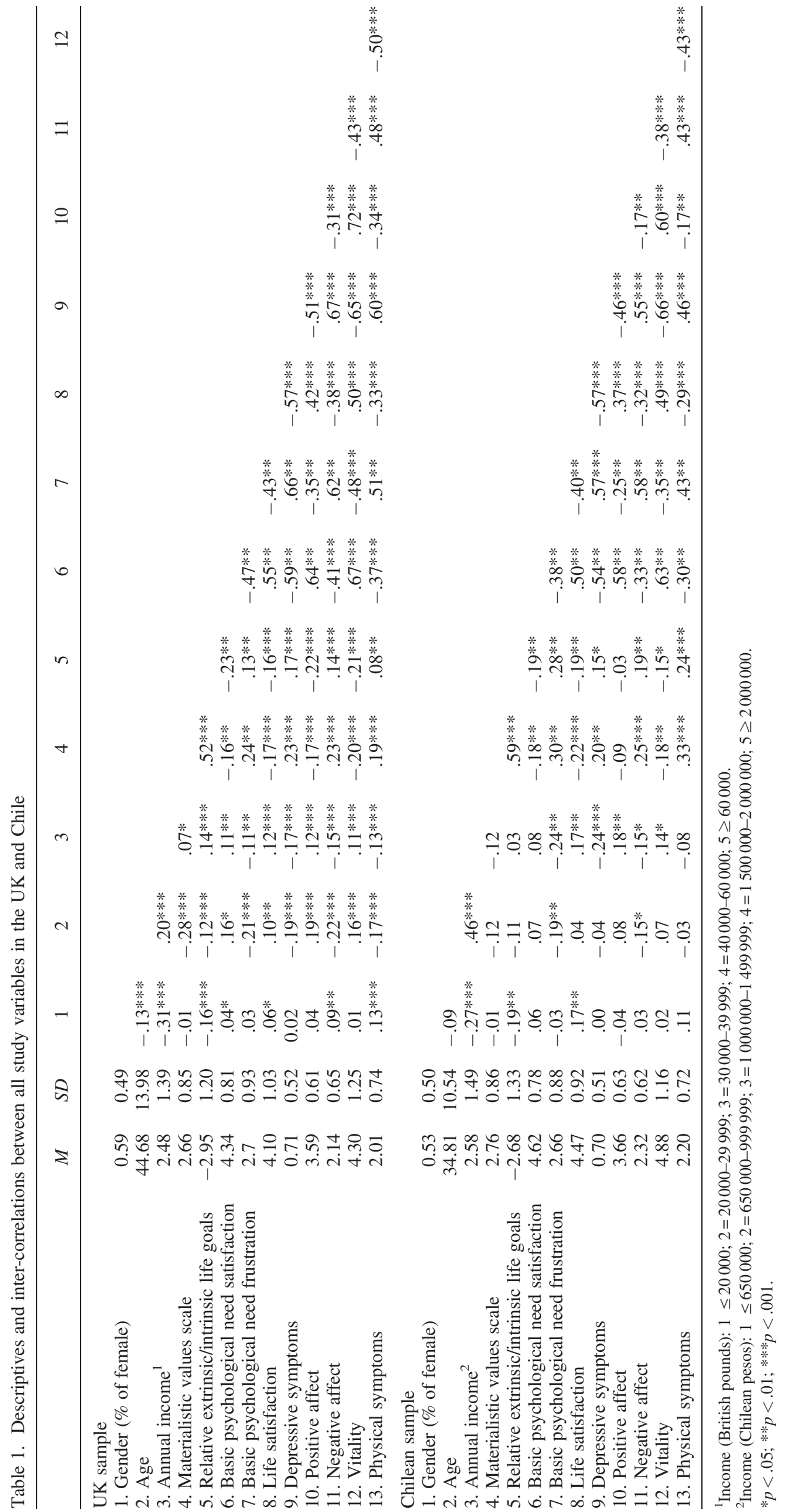


physical symptoms of ill-health, thereby using three item parcels from the General Health Questionnaire: We called this physical symptoms.

\section{Measurement model}

We developed a multigroup seven-factor measurement model for the British and Chilean samples. The results demonstrated acceptable model fit, $\chi^{2}(556)=1761.49, p<.001, C F I=0.93$, $R M S E A=0.04$. To test for metric invariance, we constrained all the factor loadings in our measurement model to be equal across the two groups, and then we compared this model to the baseline model where no constraints were imposed. The model fit remained acceptable: $\chi^{2}(575)=1826.61$, $p<.001 ; C F I=0.93 ;$ RMSEA $=0.04$. According to Cheung and Rensvold (2002), the assumption of invariance is tenable if the reduction in CFI when constraints are imposed is less than .01. Here, the reduction in CFI comfortably met this criterion $(\triangle C F I=-0.003)$. Therefore, it can be concluded that the pattern of factor loadings was invariant across countries. Hence, we maintained these constraints in all structural models reported later.

\section{Structural models}

We now tested three structural equation models to examine our different hypotheses. In initial analyses, we controlled for age, gender, and income in both samples. However, including these variables significantly affected neither the structural relationships between the latent constructs nor the results of the main predictions. Therefore, for simplicity, we report models without these additional variables.

\section{Model 1: Associations between materialism and well-being and ill-being}

To test our first hypothesis, we set up a multigroup model in which materialism was allowed to predict the four well-being outcomes (Figure 2). Initially, we estimated all structural paths freely across the two national samples. The model showed acceptable fit to the data, $\chi^{2}(335)=1310.43$, $p<.001, C F I=0.92, R M S E A=0.05$. In both countries, materialism was a significant predictor of all four well-being constructs ( $\beta$ values are shown in Figure 2). Materialism predicted lower emotional well-being and lower life satisfaction, as well as higher emotional ill-being and higher physical symptoms. $R^{2}$ values (Table 2) ranged from .03 to .07 in the UK and from .04 to .12 in Chile. In conclusion, our first hypothesis received empirical support in both countries: The endorsement of materialistic values is linked to lower wellbeing and to higher ill-being in the UK and Chile.

Then, to test whether the results differed significantly across the UK and Chilean samples, we constrained the paths from materialism to each of the four well-being constructs to be equal across the two samples. Fit statistics for

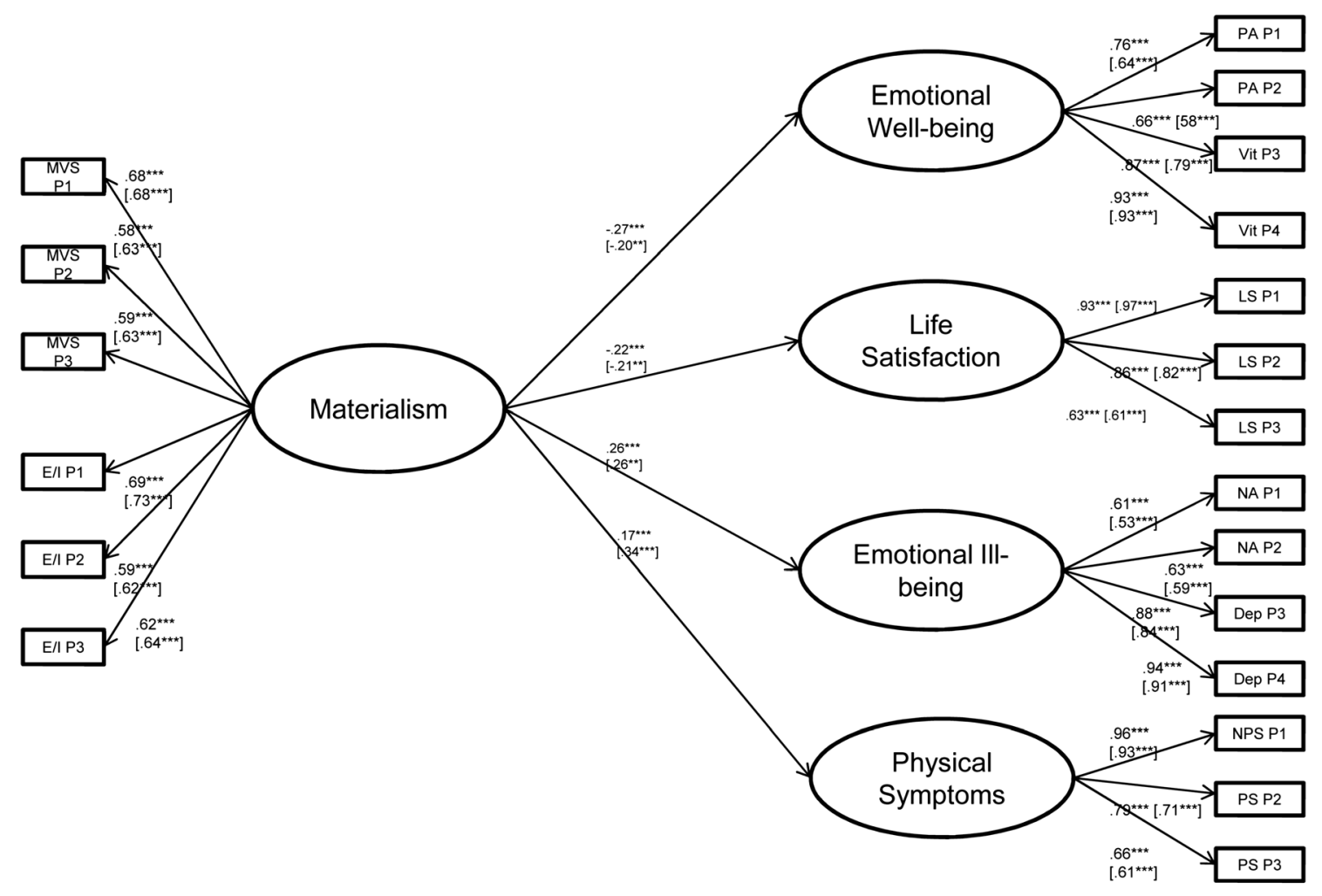

$X^{2}(335)=1310.43, p<.001 ; \mathrm{CFI}=.92 ; \mathrm{RMSEA}=.05$

Figure 2. Structural multigroup model for the associations between materialism and well-being in the UK and Chile. Coefficients shown are standardized paths. Loadings were constrained to be equal, whereas paths were estimated freely across the two national samples. Chilean coefficients are in brackets. Error terms are not shown to enhance visual clarity. MVS=materialitic values scale; $\mathrm{E} / \mathrm{I}=$ relative extrinsic-intrinsic goals; $\mathrm{Pi}=$ parcel $\mathrm{i}$; $\mathrm{PA}=$ positive affect; Vit=vitality; $\mathrm{NA}=$ negative affect; Dep $=$ depressive symptoms; $\mathrm{PS}=$ physical symptoms. $\uparrow p<.10 ; * p<.05 ; * * p<.01$; *** $p<.001$. Solid lines $=$ significant paths in both countries. 
Table 2. Modelled variance for all the constructs in the UK and Chile $R^{2}$ Model $1 \quad R^{2}$ Model $2 \quad R^{2}$ Model 3 (\%) $\quad(\%) \quad(\%)$

UK sample

1. Positive affect

and vitality

2. Satisfaction with life

3. Negative affect

and depressive symptoms

4. Physical symptoms

5. BPNS

6. BPNF

7.3

58.8

62.0

$\begin{array}{ll}4.6 & 35.8\end{array}$

43.0

$6.8 \quad 37.2$

69.0

3.0

15.8

7.6

34.0

8.0

Chilean sample

1. Positive affect

and vitality

2. Satisfaction with life

3. Negative affect

and depressive symptoms

4. Physical symptoms

5. BPNS

6. BPNF

$\begin{array}{rrr}4.1 & 59.2 & 60.1 \\ 4.4 & 33.4 & 36.7 \\ 6.8 & 41.7 & 64.4 \\ & & \\ 11.6 & 18.1 & 23.3 \\ & 1.1 & 11.0 \\ & & 17.8\end{array}$

Note: BPNS, basic psychological need satisfaction; BPNF, basic psychological need frustration.

this model showed that model fit remained acceptable, $\chi^{2}(339)=1317.72, p<<.001 ; C F I=0.92 ; R M S E A=0.05$, and there was no significant loss of fit, $\Delta \chi^{2}(4)=7.29$, $p=.12$. Therefore, there was no significant evidence that the relationship between materialism and well-being differed across the two samples.

\section{Model 2: Need satisfaction as mediator}

To test our second hypothesis, we added need satisfaction as a potential mediator in the link between materialism and personal well-being (Figure 3). Again, we initially estimated all structural paths freely across the two samples. The model showed acceptable fit indices, $\chi^{2}(447)=1582.46, p<.001 ; C F I=0.93$; $R M S E A=0.05$, with materialism relating negatively to need satisfaction in both samples. Further, in both countries, need satisfaction yielded a significant positive relation to emotional well-being and life satisfaction while being negatively related to emotional ill-being and physical symptoms. The explained variance in the well-being indicators showed a substantial improvement from Model 1 (see Table 2 for comparisons) ranging from .16 to .59 in the UK and from .18 to .59 in Chile.

In the UK, after including the mediating role of need satisfaction, the links between materialism and life satisfaction and between materialism and physical symptoms became marginally significant, whereas the links between materialism and emotional well-being and between materialism and emotional ill-being remained significant. In Chile, after including the mediating role of need satisfaction, only the link between materialism and physical symptoms remained significant. Direct paths from materialism to emotional well-being, life satisfaction, and emotional

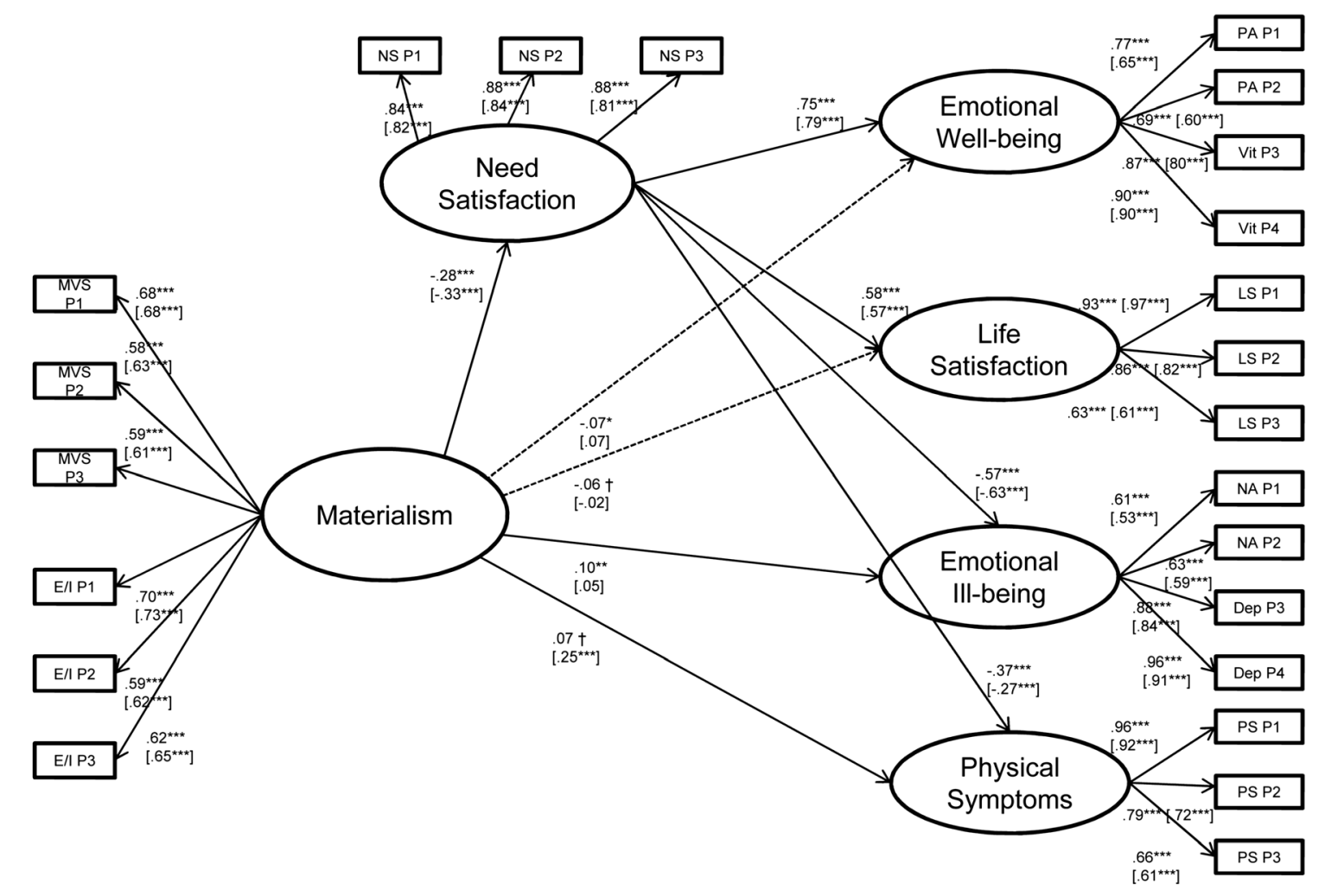

$X^{2}(447)=1582.46, p<.001 ; \mathrm{CFI}=.93 ;$ RMSEA $=.05$

Figure 3. Structural multigroup model for the associations between materialism, basic psychological need satisfaction (NS), and well-being in the UK and Chile. Coefficients shown are standardized paths. Loadings were constrained to be equal, whereas paths were estimated freely across the two national samples. Chilean coefficients are in brackets. Error terms are not shown to enhance visual clarity. MVS = materialitic values scale; E/I = extrinsic relative to intrinsic life goals; $\mathrm{Pi}=$ parcel $\mathrm{i} ; \mathrm{PA}=$ positive affect; Vit = vitality; $\mathrm{NA}=$ negative affect; Dep $=$ depressive symptoms; $\mathrm{PS}=$ physical symptoms. $\dagger p<.10 ; * p<.05 ; * * p<.01$; $*_{* *} p<.001$. Dotted lines = significant (or marginal) paths in only one country; solid lines = significant paths in both countries. 
ill-being became non-significant. All $\beta$ values are shown in Figure 3.

Therefore, it seems that in the UK, need satisfaction played a partial mediation role between materialism and all well-being constructs, providing evidence for our expectations. These results are consistent with our expectation that need frustration may need to be added to account for the remaining direct association, an issue we addressed in Model 3. In Chile, it seems that need satisfaction played a stronger mediation role, but we also expected that including need frustration would help account for the remaining direct association between materialism and physical symptoms, as well to explain additional variance in the well-being constructs.

We used Sobel tests to evaluate the significance of all indirect paths from materialism through need satisfaction to the well-being indicators in the UK and Chile. In both countries, need satisfaction significantly mediated (partially or fully) the relationships between materialism and all four well-being constructs: emotional well-being (UK: $z=-6.77, p<.001$; Chile: $z=-4.10, p<.001$ ); life satisfaction (UK: $z=-6.58, p<.05$; Chile: $z=-4.30, p<.001$ ); emotional ill-being (UK: $z=6.37$, $p<.001$; Chile: $z=4.10, p<.001$ ); and physical symptoms (UK: $z=5.90, p<.001$; Chile: $z=2.90, p<.05$ ).

Finally, to test whether the results differed significantly across the two samples, we tested a model with the structural paths constrained across samples. The model showed acceptable fit indices, $\chi^{2}(456)=1597.87, p<.001 ; C F I=0.93$; $R M S E A=0.05$, and model fit decreased only marginally, $\Delta \chi^{2}(9)=16.66, p<.10$, suggesting the paths did not differ significantly across countries.

\section{Model 3: Need satisfaction and need frustration as mediators} To test our third hypothesis, we added the basic psychological need frustration as an additional mediator, in parallel with need satisfaction (Figure 4). As before, we initially modelled all paths freely across both samples. The model showed acceptable fit indices, $\chi^{2}(575)=1826.61, p<.001 ; C F I=0.93$; $R M S E A=0.04$, and the explained variance for the well-being indicators (especially for ill-being) showed a substantial improvement from Model 2 (see Table 2 for comparisons) ranging from .34 to .69 in the UK and from .23 to .64 in Chile.

As can be noticed in Figure 4, in this model, materialism was, respectively, negatively and positively related to need satisfaction and need frustration in both samples $(\beta$ values are shown in Figure 3). Need satisfaction was a significant predictor of all the well-being constructs in both samples. Need frustration was a significant predictor of all the well-being constructs in both samples, except for the path between need frustration and emotional well-being in Chile.

In the UK sample, after including the mediating variables of both need satisfaction and need frustration, the direct paths from materialism to all four well-being constructs became

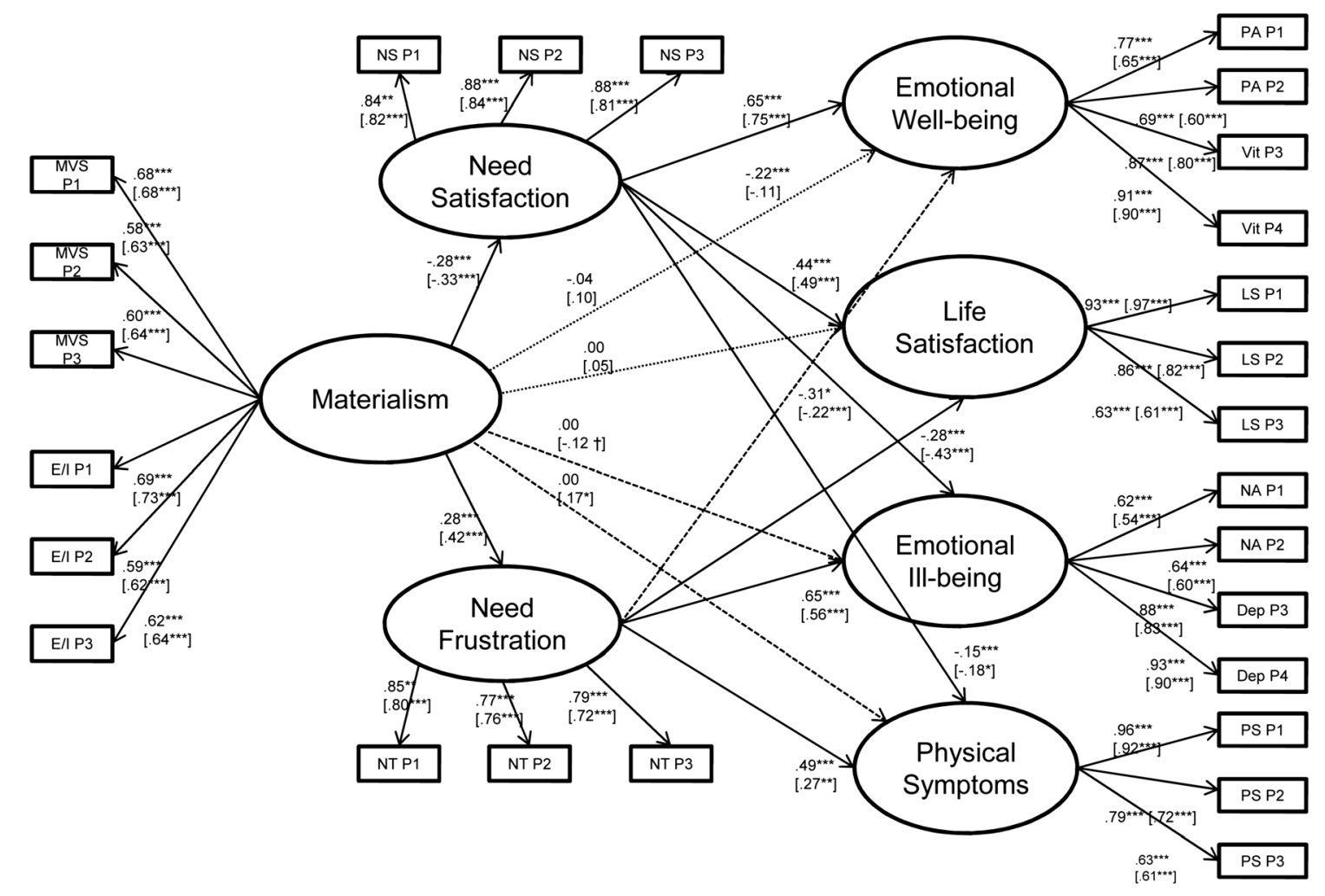

$X^{2}(575)=1826.61, p<.001 ;$ CFI $=.93 ;$ RMSEA $=.04$

Figure 4. Structural multigroup model for the associations between materialism, need satisfaction (NS), need frustration (NF), and well-being in the UK and Chile. Coefficients shown are standardized paths. Loadings were constrained to be equal, whereas paths were estimated freely across the two national samples. Chilean coefficients are in brackets. Error terms are not shown to enhance visual clarity. MVS = materialitic values scale; E/I=extrinsic relative to intrinsic life goals; LS = life satisfaction; PS = physical symptoms; $\mathrm{Pi}=$ parcel $\mathrm{i}$; PA= positive affect; Vit = vitality; $\mathrm{NA}=$ negative affect; Dep=depressive symptoms. $\dagger p$ $.10 ; * p<.05 ; * * p<.01 ; * * *<.001$. Dotted lines = significant (or marginal) paths in only one country; dashed lines = non-significant paths in both countries; solid lines $=$ significant paths in both countries. 
non-significant. In Chile, the direct paths to emotional wellbeing and life satisfaction became non-significant, whereas the direct path to emotional ill-being became marginally significant, and the direct path to negative physical symptoms remained significant (Figure 4). Thus, need satisfaction and need frustration together largely accounted for the links between materialism and all well-being outcomes in both cultures, showing the important additional role played by need frustration especially in predicting the ill-being indicators.

Sobel tests supported the mediating role of need satisfaction between materialism and all four well-being indicators in the UK and Chile: emotional well-being (UK: $z=-6.69$, $p<.001$; Chile: $z=-4.21, p<.001$ ); life satisfaction (UK: $z=-8.80, p<.05$; Chile: $z=-3.81, p<.001)$; emotional ill-being (UK: $z=7.22, p<.001$; Chile: $z=3.76, p<.001$ ); and physical symptoms (UK: $z=3.98, p<.001$; Chile: $z=2.04, p<.05)$. Sobel tests also supported the mediating role of need frustration between materialism and all the well-being constructs in the UK and Chile, except for the path between materialism and emotional well-being in Chile: emotional well-being (UK: $z=-4.97, p<.001$; Chile: $z=-1.53, p=.13$ ); life satisfaction (UK: $z=-5.42, p<.001$; Chile: $z=-2.62, p<.01$ ); emotional ill-being (UK: $z=6.50$, $p<.001$; Chile: $z=4.59, p<.001)$; and physical symptoms (UK: $z=6.20, p<.001$; Chile: $z=2.78, p<<.05$ ).

The paths shown in Figure 4 provide suggestive evidence that need satisfaction was a stronger predictor of positive outcomes, whereas need frustration was a stronger predictor of negative outcomes, but do not guarantee whether these differences were significant. Hence, we conducted a formal test of whether need satisfaction and frustration were differentially related to the four well-being constructs. To test this, we compared Model 3 with an alternative model that constrained the relative weighting of need satisfaction and frustration in predicting the four well-being outcomes to be equal, although the absolute size of these paths could vary across the four outcomes (Gausel, Leach, Vignoles, \& Brown, 2012). We specified a single latent variable (without a disturbance term) as mediating the effects between need satisfaction and need frustration and well-being. The path from need satisfaction to this latent variable was set at 1 , whereas the remaining paths were estimated freely. This alternative model showed a significantly worse fit than our original model, $\Delta \chi^{2}(6)=205.84, p<.001$, confirming that need satisfaction and frustration were differentially related to the four well-being outcomes.

Finally, to analyse whether the results differed significantly across the two samples, we now tested a model with all the structural possible paths constrained across samples. This model showed acceptable fit indices, $\chi^{2}(589)=1854.905$, $p<.001 ; C F I=0.93 ; R M S E A=0.04$. However, it showed a significant loss of fit, $\Delta \chi^{2}(14)=28.29, p<.05$. Inspection of the specific paths revealed that the direct paths between materialism and emotional well-being, materialism and physical symptoms, and need frustration and physical symptoms differed significantly across samples. Therefore, we estimated these three paths freely in each nation. Further, the results showed that this partially constrained model showed no significant loss of fit in comparison with the model with all paths estimated freely, $\Delta \chi^{2}(11)=16.53, p=.12$. Thus, it may be assumed that the remaining structural paths are not significantly different across countries. ${ }^{4}$

\section{Testing alternative causal sequences}

Our correlational design cannot answer questions about causal direction. Nonetheless, it is possible to test whether some causal sequences are more likely than others by comparing three alternative, non-nested full mediation models. We therefore compared our predicted causal sequence with two possible alternative sequences. As a baseline for comparing the alternative models, we estimated a full mediation model on the basis of our hypothesized causal sequence, omitting the direct paths from materialism to the four well-being constructs. ${ }^{5}$ This model showed an acceptable fit: $\chi^{2}(583)=1841.83$, $p<.001 ; C F I=0.93 ; R M S E A=0.04$. In the first alternative model, we examined whether need satisfaction and need frustration predict materialism, which, in turn, predicts well-being. In the second alternative model, we examined whether need satisfaction and need frustration predict well-being, which, in turn, predicts materialism. In all three models, we allowed the structural paths to vary freely across countries.

Results showed that the fit of the first alternative model was unacceptable, $\chi^{2}(591)=3144.961, p<.001 ; C F I=0.86$; $R M S E A=0.06$, whereas the second alternative model yielded, similar to our hypothesized model, an acceptable fit, $\chi^{2}(579)=1860.62, p<.001 ; C F I=0.93 ; R M S E A=0.04$. As the three models tested here were not nested, we compared them using the Akaike information criterion (AIC).

${ }^{4}$ Past research has suggested that the needs for autonomy, competence, and relatedness have unique additive effects on personal well-being (Deci \& Ryan, 2000; Sheldon \& Niemiec, 2006). Although our goal here was to distinguish between need satisfaction and need frustration as potential mediators of the link between materialism and well-being, we also tested a parallel set of models with autonomy, competence, and relatedness as separate mediators (combining satisfaction and frustration items into item parcels to measure each need) instead of need satisfaction and frustration. Following the same procedure than in our main models (e.g. constraining the loadings; modelling all paths freely across both samples), we found an acceptable model fit to the data: $\chi^{2}(719)=2072.58, p<.001 ; C F I=0.93 ; R M S E A=0.04$. In both samples, materialism predicted lower satisfaction (or greater frustration) of all three needs. However, the needs showed a more differentiated pattern of associations with the four well-being constructs. In the UK sample, autonomy and relatedness significantly mediated the relationships between materialism and all four well-being constructs (all $p<.001$ ), whereas competence significantly mediated the relationships between materialism and positive and negative emotional well-being only (both $p<.001$ ). In the Chilean sample, autonomy significantly mediated the association between materialism and physical symptoms $(p<.05)$, and relatedness significantly mediated the associations between materialism and both life satisfaction and emotional ill-being (both $p<.05$ ), whereas none of the indirect paths through competence reached significance in this sample. Nonetheless, we suspect that the failure of the remaining indirect effects to reach significance may be partly due to multicollinearity among the three needs and thus should be interpreted with caution, especially in the Chilean sample. The latent correlations between satisfactions of the three needs were all large according to Cohen's (1992) criteria, ranging from .54 to .55 in the UK and from .50 to .77 in Chile.

${ }^{5}$ To create a fair comparison across the three causal sequences that we tested, we specified all three as full mediation models. Note that it would not have been possible to compare partial mediation models, as the three partial mediation models would have included structural paths between all possible pairs of constructs, and thus, they would have been statistically indistinguishable from each other. 
AIC values for our proposed and the two alternative models were, respectively, 2079.83, 3366.961, and 2106.62. The AIC difference of 26.795 between our proposed model and the better fitting alternative model indicates that our proposed model is 658355 times as likely to minimize information loss as this alternative model, and thus our hypothesized causal sequence should be preferred to these alternatives.

These results confirm that there is better support for viewing basic need satisfaction and frustration as accounting for the relationships between materialism and well-being (hypothesized model) than there is for viewing materialism as accounting for the relationships between basic need satisfaction/frustration and well-being (alternative model 1) or for viewing well-being as accounting for the relationships between basic need satisfaction/frustration and materialism (alternative model 2). Nonetheless, we reiterate that our correlational data do not allow inferences about causal direction within each of these models. Thus, further possible sequences-where wellbeing leads to need satisfaction/frustration and need satisfaction/frustration in turn leads to materialism, or where need satisfaction/frustration has parallel effects on both materialism and well-being — would be statistically indistinguishable from our preferred model and thus equally consistent with our data.

\section{DISCUSSION}

In the current research, using relatively large samples of adults from two very different societies, and more comprehensive measures than employed for previous studies, we extended previous findings into the link between materialism and well-being in four ways.

First, supporting H1, a stronger materialistic value was associated with lower levels of psychological well-being as well as higher levels of ill-being. Our results extended earlier findings about the materialism-well-being association (Dittmar, 2008; Kasser \& Ryan, 1993, 1996, Richins \& Dawson, 1992) by showing that materialism relates not only to poorer well-being in mass-consumer societies such as the UK but also in a developing nation such as Chile (see also Dittmar \& Kapur, 2011; Ryan et al., 1999). The average size of these relationships (mean $|\beta|=.24)$ was comparable with the average disattenuated correlation of .19 between materialistic values and various indicators of lower well-being reported in the recent meta-analysis by Dittmar et al. (in press). As expected, in both samples, materialism was linked with affective, cognitive, and health dimensions of personal wellbeing, suggesting that a consumer culture orientation may have more wide-ranging negative consequences for adults than previously acknowledged.

Previous research involving the World Values Survey (e.g. Inglehart, 2000, 2008; Inglehart \& Baker, 2000) might seem to imply that materialism would be more adaptive in Chile (a less affluent nation) than in the UK (a wealthier country). However, our results do not support this view. The negative association between materialism and wellbeing was consistently found across both cultures, suggesting that the meaning attached to materialistic strivings was similarly maladaptive among both samples. We should reiterate that our measures tap into a worldview that material possessions represent a pathway to self-worth, success, and life satisfaction, whereas the World Values Survey equates materialism with a relatively broad cluster of 'survival values' some of which may be important for financial security. Indeed, in a sample of very poor Chinese rural-to-urban migrant workers, Chen, Van Assche, Vansteenkiste, Soenens, and Beyers (2013) showed that even the attainment of materialistic strivings related negatively to well-being. Yet, in this same poor sample, the experience of financial security, which was argued to stand in the service of basic survival, related positively to well-being. Thus, the exact meaning attached to financial aspirations seems to determine their relation to well-being.

Second, supporting $\mathrm{H} 2$, the association of materialistic values with lower levels of well-being and higher levels of ill-being could be accounted for by basic psychological need satisfaction. Our results showed that the higher the materialistic value orientation, the lower the need satisfaction and, as result, the less well-being and the more ill-being in the UK and in Chile. To our knowledge, this is the first research to examine either SDT or materialistic values in Chile. Searching for material rewards takes time and energy away from fulfilling basic psychological needs. Non-fulfilment of these needs leads to diminished personal well-being, causing psychological problems such as depression, negative affect, and negative physiological symptoms and also decreasing satisfaction with life and positive affect (Deci \& Ryan, 2000; Sheldon et al., 2001).

Third, extending previous work (Cordeiro et al., 2013; Sheldon, 2011; Sheldon et al., 2011; Sheldon \& Gunz, 2009; Sheldon \& Hilpert, 2012), our results supported recent claims that it is both possible and desirable to distinguish between the lack of fulfilment of basic needs and the experience of need frustration because both lower need satisfaction and higher need frustration can be detrimental to psychological well-being (Bartholomew et al., 2011; Vansteenkiste \& Ryan, 2013). In support of H3, we found that need frustration played an incremental explanatory role in the links between materialism and well-being, above and beyond the role of need satisfaction. Specifically, the higher the materialistic value orientation, the higher the need frustration and, in turn, the lower the well-being and the higher the ill-being among participants in both cultures. Therefore, these findings support recent claims stating that low need satisfaction may be different than need frustration (Vansteenkiste \& Ryan, 2013). This is a key finding, and perhaps the most novel contribution of our research. Further, consistent with recent theorizing (Bartholomew et al., 2011; Vansteenkiste \& Ryan, 2013), the satisfaction of psychological needs related primarily to well-being, whereas the frustration of the same psychological needs may especially relate to ill-being. Even though we found significant crossover paths (shown in Figure 4), results showed that need satisfaction was generally more strongly associated to positive outcomes (emotional well-being and life satisfaction) than need frustration, whereas need frustration was more strongly associated to negative outcomes (e.g. emotional ill-being and physical symptoms) than need satisfaction. This finding also extends the findings of Bartholomew et al. (2011), 
demonstrating among adult participants in two different cultures that, whereas need satisfaction is more strongly predictive of positive well-being, need frustration is more strongly associated with maladjustment and ill-being.

Fourth, and finally, we demonstrated that the negative link between materialism and well-being and the mediation of need satisfaction and need frustration did not differ, in general, across national contexts, when we compared the UK-an established mass-consumer society-with Chilea fast-growing new economy. Our main findings in the UK were replicated in the Chilean population showing that a stronger materialistic value orientation is linked to lower well-being and to higher ill-being through the mediation of need satisfaction and need frustration. In Models 2 and 3, the effect sizes of basic needs on well-being were substantially larger than the effect sizes of materialism on well-being in Model 1. This is to be expected because materialistic values are just one variable among many others that might be expected to influence well-being, whereas many different influences on well-being might be expected to have their effects through need satisfaction and need frustration (Deci \& Ryan, 2000). The fact that most of the paths in our models did not differ even in size across samples provides fairly convincing evidence for the generality of the proposed need mechanisms central to SDT, at least among the graduate populations that we sampled.

\section{LIMITATIONS AND FUTURE DIRECTIONS}

Given that our participants were all university graduates, we should be cautious about generalizing these findings to poorer and less educated groups. ${ }^{6}$ Nonetheless, several studies suggest that the link between materialism and well-being is not restricted to wealthy samples. Chen et al. (2013) found a negative association between materialism and well-being even in a poor Chinese sample. In addition, Kasser, Ryan, Zax, and Maneroff (1995) found that the most materialistic American teenagers were those who grew up in more deprived economic environments. In their recent meta-analysis, Dittmar et al. (in press) found that, although the association between materialism and personal well-being was stronger in wealthier nations, it was not moderated by personal income; nonetheless, they acknowledged that more research is needed into these processes with poorer and more heterogeneous samples.

Another limitation of our research is that method variance might be at work. For example, method effects may be driving the observed associations between the need

\footnotetext{
${ }^{6}$ Using a standard procedure (Nickerson, Schwarz, Diener, \& Kahneman, 2003) to compare incomes between countries with different purchasing power parity (World Bank, 2013, 2013), we estimated that the Chilean participants had a slightly $(7 \%)$ higher monthly personal income than the UK ones. The average income of the UK sample is located in the highest $20 \%$ of the UK national income distribution (Office for National Statistics, 2013), whereas the average income of the Chilean sample is located in the top $10 \%$ of the Chilean national income distribution (Ministerio de Desarrollo Social, 2009). Therefore, we can conclude that both samples are moderately similar in terms of socio-demographic characteristics but that they are not representative of the whole of their society. They are wealthy graduates belonging to the top level of income within their respective nations.
}

satisfaction-well-being effect and the need frustrationill-being association. This could be overcome in future work by including ratings rather than self-reports of adjustment (see Ahmad et al., 2013, for a recent example in this respect).

Finally, despite the strong evidence regarding the link between materialism and well-being, one important limitation of the present research involves its correlational design, which does not allow one to infer causality. Although our testing of alternative models allowed us to rule out some possible causal sequences, other possibilities would not be possible to distinguish statistically from our predicted sequence in the absence of longitudinal data. We have proposed here that higher materialism produces lower wellbeing due to the mediating role of need satisfaction and need frustration (after Kasser \& Ryan, 1993, 1996; Ryan \& Deci, 2000), but without longitudinal data, we cannot rule out the alternative possibility that unhappy people come to see their psychological needs as less satisfied or more frustrated, and this in turn leads them to embrace materialistic values and life goals (Dittmar, 2008). For example, research has found that when people perceive threats to their existence, they respond with various strategies. Terror management theory states that when reminded of their own mortality, people often seek out mechanisms of enhancing their self-esteem (Solomon, Greenberg, \& Pyszczynski, 1991). As a result of this threat, a common strategy for self-protection may be to endorse self-enhancing, materialistic values (Crompton \& Kasser, 2009). Following these arguments, Sheldon and Kasser (2008) found that psychological threats may increase the search for extrinsic life goals, which in turn, may negatively affect people's well-being. In other words, when people's well-being is diminished, a strategy could be to engage in a materialistic value orientation.

Hence, longitudinal research is needed to evaluate the directionality of the link between materialism and well-being over time. Such research may also help to shed light on the mechanisms that intervene in the relation between extrinsic, relative to intrinsic, goals and experiences of need satisfaction and need frustration. Although it is maintained within SDT that intrinsic goals create opportunities for need satisfaction, whereas extrinsic goal contents interfere with need satisfaction and may even elicit need frustration, the intervening processes in these associations have received little attention (Vansteenkiste et al., 2008). Thus, apart from the more global mechanisms of need satisfaction and need frustration, more specific mechanisms should be examined. For instance, extrinsic and intrinsic goal contents may, respectively, preclude or foster task absorption, which, respectively, hampers or contributes to competence development (Vansteenkiste et al., 2004). Further, a focus on extrinsic goals may relate to more frequent and different forms of interpersonal comparisons (Sebire, Standage, Gillison, \& Vansteenkiste, 2013), which may be socially alienating, stressful, and produce a sense of incompetence. Gaining more exact insight in these intervening processes may help to set up and test intervention programmes in which adults are trained to become resilient against the negative effects of being exposed to a mass-consumer culture. 


\section{REFERENCES}

Ahmad, I., Vansteenkiste, M., \& Soenens, B. (2013). The relations of Arab Jordanian adolescents' perceived maternal parenting to teacher-rated adjustment and problems: The intervening role of perceived need satisfaction. Developmental Psychology, 49, 177-183

Banerjee, R. \& Dittmar, H. (2008). Individual differences in children's materialism: The role of peer relations. Personality and Social Psychology Bulletin, 34, 17-31. DOI: 10.1177/ 0146167207309196.

Bartholomew, K. J., Ntoumanis, N., Ryan, R. M., Bosch, J. A., \& Thøgersen-Ntoumani, C. (2011). Self-determination theory and diminished functioning: The role of interpersonal control and psychological need thwarting. Personality and Social Psychology Bulletin, 37, 1459-1473. DOI:10.1177/0146167211413125.

Belk, R. W. (1985). Materialism: Trait aspects of living in the material world. Journal of Consumer Research, 12, 265-280. DOI: $10.1086 / 208515$.

Brislin, R. W. (1970). Back-translation for cross-cultural research. Journal of Cross-Cultural Psychology, 1, 185-216. DOI: 10.1177/135910457000100301.

Burroughs, J. E., \& Rindfleisch, A. (2002). Materialism and wellbeing: A conflicting values perspective. Journal of Consumer Research, 29, 348-370. DOI: 10.1086/344429.

Chen, B., Van Assche, J., Vansteenkiste, M., Soenens, B., \& Beyers, W. (2013). Does psychological need satisfaction matter when financial or environmental safety is at risk? Manuscript submitted for publication, University of Ghent, Belgium.

Cheung, G. W., \& Rensvold, R. B. (2002). Evaluating goodness-offit indexes for testing measurement invariance. Structural Equation Modeling, 9, 233-255. DOI: 10.1207/s15328007sem0902_5.

Chirkov, V. I., Ryan, R. M., \& Sheldon, K. M. (2011). Human autonomy in cross-cultural contexts: Perspectives on the psychology of agency, freedom, and well-being. Dordrecht: Springer.

Chirkov, V. I., Ryan, R. M., \& Willness, C. (2005). Cultural context and psychological needs in Canada and Brazil: Testing a selfdetermination approach to the internalization of cultural practices, identity, and well-being. Journal of Cross-Cultural Psychology, 36, 423-443. DOI: 10.1177/0022022105275960.

Christopher, A. N., Kuo, S. V., Abraham, K. M., Noel, L. W., \& Linz, H. E. (2004). Materialism and affective well-being: The role of social support. Personality and Individual Differences, 37, 463-470. DOI: 10.1016/j.paid.2003.09.015.

Christopher, A. N., \& Schlenker, B. R. (2004). Materialism and affect: The role of self presentational concerns. Journal of Social and Clinical Psychology, 23, 260-272. DOI: 10.1521/ jscp.23.2.260.31022.

Church, A. T., Katigbak, M. S., Locke, K. D., Zhang, H., Shen, J., de Jesús Vargas-Flores, J., ... \& Ching, C. M. (2013). Need satisfaction and well-being testing self-determination theory in eight cultures. Journal of Cross-Cultural Psychology, 44, 507-534.

Cohen, J. (1992). A power primer. Psychological Bulletin, 112, 155.

Cordeiro, P., Paixão, M. P., Lenes, W., \& Silva, J. (2013). The Portuguese version of the Balanced Measure of Psychological Needs Scale: Factor structure, validity and relations to perceived parenting. Manuscript submitted for publication, University of Coimbra, Portugal.

Crompton, T., \& Kasser, T. (2009). Meeting environmental challenges: The role of human identity. Retrieved from the WWF website: www.wwf.org.uk.

Deci, E. L., \& Ryan, R. M. (2000). The "what" and "why" of goal pursuits: Human needs and the self-determination of behavior. Psychological Inquiry, 11, 227-268. DOI: 10.1207/ S15327965PLI1104_01.

Deci, E. L., Ryan, R. M., Gagné, M., Leone, D. R., Usunov, J., \& Kornazheva, B. P. (2001). Need satisfaction, motivation, and well-being in the work organizations of a former eastern bloc country: A cross-cultural study of self-determination. Personality and Social Psychology Bulletin, 27, 930-942.
Diener, E. (1984). Subjective well-being. Psychological Bulletin, 95, 542-575. DOI: 10.1037/0033-2909.95.3.542.

Diener, R., Emmons, A. , Larsen, R., \& Griffin, S. (1985). The satisfaction with life scale. Journal of Personality Assessment, 49, 71-75. DOI: 10.1207/s15327752jpa4901_13.

Diener, E., \& Tay, L. (2012). A scientific review of the remarkable benefits of happiness for successful and healthy living. Report of the Well-Being Working Group, Royal Government of Bhutan: Report to the United Nations General Assembly, Well-Being and Happiness: A New Development Paradigm, UN, NY.

Dittmar, H. (2008). Consumer culture, identity, and well-being: The search for the "good life" and the "body perfect". Hove, UK: Psychology Press.

Dittmar, H., Bond, R., Kasser, T., \& Hurst, M. (in press). The relationship between materialism and personal well-being: A meta-analysis. Journal of Personality and Social Psychology.

Dittmar, H., \& Kapur, P. (2011). Consumerism and well-being in India and the UK: Identity projection and emotion regulation as underlying psychological processes. Psychological Studies, 56, 71-85. DOI: 10.1007/s12646-011-0065-2.

Duriez, B., Vansteenkiste, M., Soenens, B., \& De Witte, H. (2007). The social costs of extrinsic relative to intrinsic goal pursuits: Their relation with social dominance and racial and ethnic prejudice. Journal of Personality, 75, 757-782. DOI: 10.1111/j.14676494.2007.00456.x.

Gallagher, M. W., Lopez, S. J., \& Preacher, K. J. (2009). The hierarchical structure of well being. Journal of personality, 77 , 1025-1050. DOI: 10.1111/j.1467-6494.2009.00573.x.

Gatersleben, B., Jackson, T., Meadows, J., Soto, E., \& Yan, Y. (2012). Materialism and environmentalism. Exploring the views of young adults in the UK, Spain and China. Manuscript submitted for publication, University of Surrey, UK.

Gausel, N., Leach, C. W., Vignoles, V. L., \& Brown, R. (2012). Defend or repair? Explaining responses to in-group moral failure by disentangling feelings of shame, rejection, and inferiority. Journal of personality and social psychology, 102, 941.

Goldberg, D. P., Gater, R., Sartorius, N., Ustun, T. B., Piccinelli, M., Gureje, O., \& Rutter, C. (1997). The validity of two versions of the GHQ in the WHO study of mental illness in general health care. Psychological medicine, 27, 191-197.

Hu, L., \& Bentler, P. M. (1999). Cutoff criteria for fit indexes in covariance structure analysis: Conventional criteria versus new alternatives. Structural Equation Modeling, 6, 1-55. DOI: 10.1080/10705519909540118.

Inglehart, R. (2000). Globalization and postmodern values. Washington Quarterly, 23, 215-228. DOI: 10.1162/016366000560665.

Inglehart, R. F. (2008). Changing values among western publics from 1970 to 2006. West European Politics, 31, 130-146. DOI: 10.1080/01402380701834747.

Inglehart, R., \& Baker, W. E. (2000). Modernization, cultural change, and the persistence of traditional values. American sociological review, 65, 19-51. DOI:10.2307/2657288.

Kasser, T., \& Ahuvia, A. (2002). Materialistic values and wellbeing in business students. European Journal of Social Psychology, 32, 137-146. DOI: 10.1002/ejsp.85.

Kasser, T., \& Kanner, A. D. (2004). Psychology and consumer culture: The struggle for a good life in a materialistic world (Vol. 297). Washington, DC: APA.

Kasser, T. \& Ryan, R. M. (1993). A dark side of the American dream: Correlates of financial success as a central life aspiration. Journal of Personality and Social Psychology, 65, 410-422. DOI: $10.1037 / 0022-3514.65 .2 .410$.

Kasser, T., \& Ryan, R. M. (1996). Further examining the American dream: Differential correlates of intrinsic and extrinsic goals. Personality and Social Psychology Bulletin, 22, 280-287. DOI: 10.1177/0146167296223006.

Kasser, T., Ryan, R. M., Zax, M., \& Sameroff, A. J. (1995). The relations of maternal and social environments to late adolescents' materialistic and prosocial values. Developmental psychology, 31, 907-914. 
Keyes, C. L., Shmotkin, D., \& Ryff, C. D. (2002). Optimizing well-being: The empirical encounter of two traditions. Journal of personality and social psychology, 82, 207-222. DOI: 10.1037/0022-3514.82.6.1007.

Kline, R. B. (2005). Principles and practice of structural equation modelling. (2nd Ed.). New York: Guilford Press.

Little, T., Cunningham, W., \& Shahar, G. (2002). To parcel or not to parcel: Exploring the question, weighing the merits. Structural Equation Modelling, 9, 151-173. DOI: 10.1207/ S15328007SEM0902_1.

Ministerio de Desarrollo Social (2009). Distribución del Ingreso en Chile, Encuesta Casen. Retrieved from the Ministerio de Desarrollo Social website http://www.ons.gov.uk/ons/dcp171778_317365.pdf.

Mouratidis, A., Vansteenkiste, M., Lens, W., Michou, A., \& Soenens, B. (2013). Within person configurations and temporal relations of personal and perceived parent-promoted aspirations to school correlates among adolescents. Journal of Educational Psychology, 105, 895-910. DOI: 10.1037/a0032838.

Nickerson, C., Schwarz, N., Diener, E., \& Kahneman, D. (2003). Zeroing in on the dark side of the American dream a closer look at the negative consequences of the goal for financial success. Psychological Science, 14, 531-536. DOI: 10.1046/j.09567976.2003.psci_1461.x.

OECD (2013). OECD Factbook 2013: Economic, Environmental and Social Statistics. Retrieved on 08/09/2013 from the OECD website: http://www.oecd-ilibrary.org/economics/oecd-factbook2013_factbook-2013-en.

Office for National Statistics (2013). The Effects of Taxes and Benefits on Household Income, 2011/12. Retrieved from the Office for National Statistics website http://www.ons.gov.uk/ons/ dcp171778_317365.pdf.

Oishi, S., Diener, E. F., Lucas, R. E., \& Suh, E. M. (1999). Crosscultural variations in predictors of life satisfaction: Perspectives from needs and values. Personality and social psychology bulletin, 25, 980-990.

Radloff, L. S. (1977). The CES-D scale a self-report depression scale for research in the general population. Applied psychological measurement, 1, 385-401. DOI: 10.1177/014662167700100306.

Richins, M. (2004). The material values scale: Measurement properties and development of a short form. Journal of Consumer Research, 31, 209-219. DOI: 10.1086/383436.

Richins, M., \& Dawson, S. (1992). A consumer values orientation for materialism and its measurement-Scale development and validation. Journal of Consumer Research, 19, 303-316. DOI: 10.1086/209304.

Ryan, R. M., Chirkov, V. I., Little, T. D., Sheldon, K. M., Timoshina, E., \& Deci, E. L. (1999). The American dream in Russia: Extrinsic aspirations and well-being in two cultures. Personality and Social Psychology Bulletin, 25, 1509-1524.

Ryan, R. M., \& Deci, E. L. (2000). Self-determination theory and the facilitation of intrinsic motivation, social development, and well-being. American Psychologist, 55, 68-78. DOI: 10.1037/ 0003-066X.55.1.68.

Ryan, R. M., \& Deci, E. L. (2001). On happiness and human potentials: A review of research on hedonic and eudaimonic well-being. Annual Review of Psychology, 52, 141-166. DOI: 10.1146/annurev.psych.52.1.141.

Ryan, R. M., \& Frederick, C. (1997). On energy, personality, and health: Subjective vitality as a dynamic reflection of well-being. Journal of personality, 65, 529-565. DOI:10.5502/ijw.v1i3.1.

Ryff, C. D. (1989). Happiness is everything, or is it? Explorations on the meaning of psychological well-being. Journal of Personality and Social Psychology, 57, 1069-1081.

SBIF (2011). Productos. Retrieved from the Superintendencia de Bancos e Instituciones Financieras website: http://www.sbif.cl/ sbifweb/servlet $/$ Portada?indice $=0.0$.

Schaefer, A. D., Hermans C. M., \& Parker R. S. (2004). A crosscultural exploration of materialism in adolescents. International Journal of Consumer Studies, 28, 399-411. DOI: 10.1111/ j.1470-6431.2004.00395.x.
Schwartz, S. H. (1992). Universals in the content and structure of values: Theoretical advances and empirical tests in 20 countries. Advances in experimental social psychology, 25, 1-65.

Sebire, S., Standage, M., Gillison, F., \& Vansteenkiste, M. (2013). "Coveting thy neighbour's legs": A qualitative study of exercisers' experiences of intrinsic and extrinsic goal pursuit. Journal of Sport \& Exercise Psychology, 35, 308-321.

Sebire, S. J., Standage, M., \& Vansteenkiste, M. (2009). Examining intrinsic versus extrinsic exercise goals: Cognitive, affective, and behavioral outcomes. Journal of Sport \& Exercise Psychology, 31, 189-210.

Sheldon, K. M. (2011). Integrating behavioral-motive and experiential-requirement perspectives on psychological needs: A two process model. Psychological review, 118, 552. DOI: 10.1037/a0024758.

Sheldon, K. M., Abad, N., \& Hinsch, C. (2011). A two-process view of Facebook use and relatedness need-satisfaction: Disconnection drives use, and connection rewards it. Psychology of Popular Media Culture, 1, 2-15. DOI: 10.1037/2160-4134.1.s.2.

Sheldon, K. M., Elliot, A. J., Kim, Y., \& Kasser, T. (2001). What is satisfying about satisfying events? Testing 10 candidate psychological needs. Journal of Personality and Social Psychology, 80, 325-339. DOI: 10.1037//0022-3514.80.2.325.

Solomon, S., Greenberg, J., \& Pyszczynski, T. (1991). A terrormanagement theory of social behaviour: The psychological functions of self-esteem and cultural worldviews. San Diego: Academic Press.

Sheldon, K. M., \& Gunz, A. (2009). Psychological needs as basic motives, not just experiential requirements. Journal of Personality, 77, 1467-1492. DOI: 10.1111/j.1467-6494.2009.00589.x.

Sheldon, K. M., \& Hilpert, J. C. (2012). The balanced measure of psychological needs (BMPN) scale: An alternative domain general measure of need satisfaction. Motivation and Emotion, 36, 439-451. DOI:10.1007/s11031-012-9279-4.

Sheldon, K. M., \& Kasser, T. (2008). Psychological threat and extrinsic goal striving. Motivation and Emotion, 32, 37-45.

Sheldon, K. M., \& Niemiec, C. P. (2006). It's not just the amount that counts: Balanced need satisfaction also affects well-being. Journal of personality and social psychology, 91, 331. DOI: 10.1037/0022-3514.91.2.331.

Stebbings, J., Taylor, I. M., Spray, C. M., \& Ntoumanis, N. (2012). Antecedents of perceived coach interpersonal behaviors: The coaching environment and coach psychological well-and illbeing. Journal of Sport \& Exercise Psychology, 34, 481-502.

Tang, T. L. P., Kim, J. K., \& Tang, T. L. N. (2002). Endorsement of the money ethic, income, and life satisfaction: A comparison of full-time employees, part-time employees, and non-employed university students. Journal of Managerial Psychology, 17, 442-467. DOI: 10.1108/02683940210439388.

Tang, T. L. P., Tang, D. S. H., \& Luna-Arocas, R. (2005). Money profiles: The love of money, attitudes, and needs. Personnel Review, 34, 603-618. DOI: 10.1108/00483480510612549.

Taylor, I. M., \& Lonsdale, C. (2010). Cultural differences in the relationships among autonomy support, psychological need satisfaction, subjective vitality, and effort in British and Chinese physical education. Journal of Sport \& Exercise Psychology, 32, 655-673.

Thøgersen-Ntoumani, C., Ntoumanis, N., \& Nikitaras, N. (2010). Unhealthy weight control behaviours in adolescent girls: A process model based on self-determination theory. Psychology and Health, 25, 535-550. DOI: 10.1080/08870440902783628.

Thompson, E. R. (2007). Development and validation of an internationally reliable short form of the positive and negative affect schedule (PANAS). Journal of Cross-Cultural Psychology, 38, 227-242. DOI: 10.1177/0022022106297301.

Twenge, J. M. (2010). A review of the empirical evidence on generational differences in work attitudes. Journal of Business and Psychology, 25, 201-210.

Twenge, J. M., Campbell, W. K., \& Freeman, E. C. (2012). Generational differences in young adults' life goals, concern for others, 
and civic orientation, 1966-2009. Journal of Personality and Social Psychology, 102, 1045-1062. DOI: 10.1037/a0027408.

Twenge, J. M., \& Kasser, T. (2013). Generational changes in materialism and work centrality, 1976-2007 associations with temporal changes in societal insecurity and materialistic role modeling. Retrieved from the Personality and Social Psychology Bulletin website: http://psp.sagepub.com/content/early/2013/05/ 01/0146167213484586. DOI: 10.1177/0146167213484586.

United Nations Development Program (2010). Human Development Report: The real wealth of nations: Pathways to Human Development. Retrieved from the United Nations website: http://hdr.undp.org/en/media/HDR_2010_EN_Complete_reprint. pdf on 14/08/2011.

Van Hiel, A., Cornelis, I., \& Roets, A. (2010). To have or to be? A comparison of materialism-based theories and self-determination theory as explanatory frameworks of prejudice. Journal of personality, 78, 1037-1070. DOI: 10.1111/j.1467-6494.2010.00642.x.

Vansteenkiste, M., Duriez, B., Simons, J., \& Soenens, B. (2006). Materialistic values and well-being among business students: Further evidence of their detrimental effect. Journal of Applied Social Psychology, 36, 2892-2908.

Vansteenkiste, M., Neyrinck, B., Niemiec, C. P., Soenens, B., Witte, H., \& Broeck, A. (2007). On the relations among work value orientations, psychological need satisfaction and job outcomes: A self-determination theory approach. Journal of Occupational and Organizational Psychology, 80, 251-277. DOI: 10.1348/096317906X111024.

Vansteenkiste, M., Niemiec, C. P., \& Soenens, B. (2010). The development of the five minitheories of self-determination theory: A historical overview, emerging trends and future directions. In T. Urdan, \& S. Karabenick (Eds.), Advances in motivation and achievement, vol. 16: The decade ahead (pp. 105-166). UK: Emerald Publishing. DOI:10.1108/S0749-7423(2010)000016A007.

Vansteenkiste, M., \& Ryan, R. M. (2013). On psychological growth and vulnerability: Basic psychological need satisfaction and need frustration as a unifying principle. Journal of Psychotherapy Integration, 3, 263-280.

Vansteenkiste, M., Simons, J., Lens, W., Soenens, B., Matos, L., \& Lacante, M. (2004). "Less is sometimes more": Goal-content matters. Journal of Educational Psychology, 96, 755-764.

Vansteenkiste, M., Soenens, B., \& Duriez, B. (2008). Presenting a positive alternative to materialistic strivings and the thin-ideal: Understanding the effects of extrinsic relative to intrinsic goal pursuits. In S. J. Lopez (Ed.), Positive psychology: Exploring the best in people (Vol. 4, pp. 57-86). Westport, CT: Greenwood Publishing Company.

Verstuyf, J., Vansteenkiste, M., \& Soenens, B. (2012). Eating regulation and bulimic symptoms: The differential correlates of health-focused and appearance-focused eating regulation. Body Image , 9, 108-117. DOI: 10.1016/j.bodyim.

Verstuyf, J., Vansteenkiste, M., Soenens, B., Boone, L., \& Mouratidis, T. (2013). Daily ups and downs in women's binge eating symptoms: The role of basic psychological needs, general self-control and emotional eating. Journal of Social and Clinical Psychology, 32, 335-361.

Waterman, A. S., Schwartz, S. J., Zamboanga, B. L., Ravert, R. D., Williams, M. K., Agocha, V. B., Kim, S. Y., \& Donnellan, M. B. (2010). The questionnaire for eudaimonic well-being: Psychometric properties, demographic comparisons, and evidence of validity. Journal of Positive Psychology, 5, 41-61.

World Bank (2013a). PPP conversion factor, private consumption (LCU per international \$). Retrieved on 08/09/2013 from the World Bank website: http://data.worldbank.org/indicator/PA. NUS.PRVT.PP.

World Bank (2013b). GDP per capita, PPP (current international \$). Retrieved on 08/09/2013 from the World Bank website: http://data. worldbank.org/indicator/NY.GDP.PCAP.PP.CD.

Williams, G. C., Cox, E. M., Hedberg, V., \& Deci, E. L. (2000). Extrinsic life goals and health risk behaviors in adolescents. Journal of Applied Social Psychology, 30, 1756-1771. 